\title{
Alterações eletrofisiológicas na esquizofrenia
}

\author{
Luis FH Basile
}

Laboratório de Neurociências (LIM-27). Departamento de Psiquiatria da FMUSP

Por definição, alterações no eletroencefalograma (EEG) convencional excluem o diagnóstico de esquizofrenia. Entendese por EEG convencional o estudo, por inspeção visual, de diferenças de potencial entre pares de eletrodos afixados ao couro cabeludo. No entanto, a busca continuada de marcadores fisiopatológicos das esquizofrenias motivou o uso de novas técnicas, na esperança de uma maior sensibilidade a possíveis alterações sutis do funcionamento do encéfalo. Dentre tais técnicas destacam-se os métodos quantitativos e, especialmente, a computação de médias de voltagens sincronizadas com eventos definidos no tempo, conhecidas como potenciais evocados e provocados.

\section{EEGquantitativo}

O método quantitativo mais comumente usado é a análise da distribuição de potência elétrica em faixas de frequiência, ou composição espectral do EEG. Trechos do EEG (variações de voltagens no tempo) podem ser transformados no equivalente matemático de potência elétrica (proporcional ao quadrado da voltagem) em função de freqüência. Assim, grupos de pacientes esquizofrênicos podem ser comparados com grupos de indivíduos saudáveis quanto à potência total em determinadas faixas. Um estudo, utilizando 14 eletrodos em relação a um ponto comum na cabeça (montagem monopolar), mostrou um aumento global de potência no EEG de repouso no grupo de esquizofrênicos em relação ao grupo controle, em especial nas baixas frequiências e em eletrodos frontais esquerdos (bandas delta e teta, de 1 a $8 \mathrm{~Hz}$ ). ${ }^{1} \mathrm{O}$ achado de um aumento de potência nas faixas delta e teta foi corroborado por um estudo mais recente com grande número de pacientes. ${ }^{2}$ Esses estudos apóiam a idéia de hipofunção do córtex frontal, que nesse caso manifesta-se como um aumento de regularidade ou ritmicidade da atividade elétrica de repouso. Deve-se, porém, manter a ressalva com respeito à topografia dos achados: um achado válido para determinado eletrodo frontal em relação à mastóide, por exemplo, não prova definitivamente que o córtex subjacente seja o foco da alteração.

\section{Potenciais evocados sensitivos}

A segunda técnica, a dos potenciais evocados e provocados, permite o estudo de relações entre variações elétricas médias e eventos definidos no tempo, incluindo estímulos sensoriais, comportamentos motores e estados psíquicos específicos. Assim, uma gama virtualmente infinita de situações experi- mentais pode ser testada, em particular para a verificação de alterações do funcionamento encefálico em esquizofrênicos. Os potenciais evocados sensitivos, relativamente mais conhecidos, foram os mais utilizados na pesquisa psiquiátrica. Tais potenciais são computados como médias entre trechos de EEG obtidos durante muitas repetições da apresentação de determinado tipo de estímulo. Os trechos de EEG têm como tempo zero o início da duração dos estímulos, e as médias resultantes terão um número de deflexões de voltagem positiva e negativa dependente da modalidade sensorial, freqüência e intensidade de estimulação, e diversos outros fatores, incluindo atenção e implicações comportamentais da detecção dos estímulos. Usualmente, a direção de uma deflexão e sua latência em relação ao início dos estímulos definem determinado potencial evocado. Assim, por exemplo, P50 auditivo refere-se a uma deflexão positiva do potencial médio obtido pela repetição de cliques sonoros, com pico de voltagem de aproximadamente 50 milissegundos após o início dos cliques. Dentre os achados eletrofisiológicos na esquizofrenia, o mais robusto refere-se justamente ao P50. ${ }^{3}$ Indivíduos saudáveis, quando estimulados por pares de cliques, separados por meio segundo, apresentam o segundo P50 relativamente suprimido em relação ao primeiro. Pacientes esquizofrênicos não apresentam tal supressão. Apesar de diversos autores considerarem a não supressão do P50 uma indicação de supersensibilidade dos pacientes ou ausência de mecanismos inibitórios e seletivos relacionados à atenção, é possível que o fenômeno reflita apenas uma variabilidade aumentada na latência do primeiro P50 obtido nos pacientes. ${ }^{4}$ Diversas alterações de amplitude e latência de potenciais evocados auditivos, visuais e somatosensitivos já foram relatadas na literatura, mas tais achados não são livres de controvérsias, e prefere-se aguardar replicações suficientes.

\section{Potenciais evocados endógenos}

Enquanto os estados de vigília e integridade das vias sensoriais são suficientes para a obtenção dos potenciais evocados sensitivos, o uso de tarefas explícitas, que exijam atenção voluntária, discriminação entre estímulos e antecipação de eventos, modula os potenciais sensoriais e gera novos componentes. Tais componentes são os potenciais evocados endógenos e potenciais provocados. Uma classe de potenciais endógenos que surge apenas quando há atenção aos estímulos é a do P300. ${ }^{5}$ A tarefa prototípica, em que se verifica um P300 auditivo, consiste na contagem de tons raros $(2.000 \mathrm{~Hz})$ dentre uma série 
de tons de $1.000 \mathrm{~Hz}$. Tal classe inclui, no entanto, potenciais que atingem pico até 700 milissegundos após um estímulo (visual complexo, por exemplo). Sabe-se que esses potenciais aumentam em amplitude e latência com a raridade, complexidade e significado comportamental dos estímulos, ${ }^{5}$ e são gerados por um conjunto de áreas corticais posteriores, modalidade-específicas e inespecíficas, incluindo a formação hipocampal. ${ }^{6}$ Tanto latência aumentada como amplitudes reduzidas de P300 auditivos, visuais e somatossensitivos já foram observadas em grupos de esquizofrênicos..$^{7-11}$ Tais alterações são especialmente válidas para pacientes não paranóides ${ }^{8}$ e parecem ser marcadores independentes de medicação ou estágio da doença.

Conforme afirma-se acima, a atenção voluntária modula em amplitude e latências diversos potenciais evocados sensitivos. De acordo com isso, uma técnica interessante de análise de EEG baseia-se na subtração entre potenciais médios, obtidos durante situações envolvendo atenção e relevância de estímulos, e os potenciais obtidos durante estimulação passiva. Normalmente, uma negativação adicional dos potenciais evocados associa-se à condição de atenção. Ou seja, potenciais resultantes da subtração incluem picos negativos de voltagem, iniciando mesmo antes de 100 milissegundos após os estímulos. Um exemplo é a acentuação dos N200 visual ou auditivo (deflexão negativa de latência próxima a 200 milissegundos após estímulos), que interessantemente está muito reduzida e atrasada em esquizofrênicos. ${ }^{12}$ Outros potenciais de subtração ocorrem em situações de quebra de expectativas, seja quando um estímulo anômalo interrompe uma seqüência regular ("mismatch negativity", MMN) ou quando uma palavra anômala quebra o sentido de uma sentença familiar (N400). Alguns estudos recentes em grupos de pacientes esquizofrênicos mostram que a MMN é muito reduzida. ${ }^{13} \mathrm{Cu}$ riosamente, apesar de diferentes relatos na literatura sobre a amplitude do N400 em pacientes, esse potencial, tido como índice de perplexidade ou "ativação" semântica, parece consistentemente atrasado e persistente nos pacientes. ${ }^{14}$

\section{Potenciais provocados}

Finalmente, deve-se considerar os potenciais provocados, cujo representante mais relevante é a classe das Variações Contingentes Negativas (VCNs). As VCNs são potenciais negativos lentos, com máximo usualmente em regiões centro-frontais (em relação às mastóides), que surgem na antecipação de eventos que ocorrem em intervalos de tempo fixos. ${ }^{15}$ As VCNs são particularmente interessantes por seus correlatos psicológicos, especialmente por serem geradas em diversos córtices associativos, e por constituírem praticamente os únicos indicadores eletrofisiológicos de atividade no córtex frontal. ${ }^{*}$ Estudos com grupos de pacientes esquizofrênicos mostraram uma redução de amplitude de VCNs convencionais (obtidas durante tarefas de mera temporalização de respostas motoras), o que também parece um marcador da doença, independente de medicação ou estágio evolutivo. ${ }^{16-18}$
Diversos estudos dentre os aqui discutidos tomam conclusões topográficas que não se justificam pelo pequeno número de eletrodos usados e pela referência dos mesmos a eletrodos que não podem ser considerados neutros. O ideal de todos os métodos de análise deve ser a conciliação de achados sobre o funcionamento encefálico com as diversas situações psicológicas controladas experimentalmente. Para se efetuar tais correlações anatomofuncionais, é necessário que um grande número de eletrodos seja usado (>100), referenciados à média de potencial entre eletrodos, e que os dados de superfície sejam traduzidos em correntes intracranianas por programas de reconstrução apenas recentemente disponíveis. Iniciou-se no Laboratório de Neurociências um experimento com tal metodologia em pacientes esquizofrênicos e indivíduos controle, usando uma tarefa visual de memorização de pares de estímulos (verbais, espaciais ou pictóricos). Analisaram-se diversos tipos de potenciais, incluindo os evocados visuais, P300 visual e VCNs, para os diferentes tipos de estímulos e durante duas condições: memorização e evocação. Durante a evocação, usaram-se estímulos interessantes, que indicam acerto ou erro (feedback) para cada par. Na etapa preliminar de análise, computaram-se médias de voltagem entre indivíduos de cada grupo (19 controle e 12 pacientes). Dentre os primeiros achados notáveis, válidos para as médias do grupo de esquizofrênicos em relação ao controle, estão uma grande redução da razão sinal/ruído de todos os potenciais (amplitude do potencial dividida por atividade de fundo), especialmente para o P300 pictórico; diminuição de praticamente todos os potenciais evocados; aumento de potência das VCNs de memorização; e topografia alterada e menor definição dos extremos de voltagem para a VCN de antecipação de feedback. Ademais, a análise de geradores intracranianos das VCNs mostrou uma maior dispersão de correntes corticais em todas as condições para os pacientes. Os últimos dois achados podem indicar maior heterogeneidade no grupo de pacientes, o que concordaria com resultados obtidos por outras metodologias.

Usar-se-ão os resultados obtidos para índices médios de grupo como guia para a detecção de anomalias individuais, uma vez que se realizou análise de geradores intracranianos para os dados de cada indivíduo. Será então possível agrupar pacientes de acordo com marcadores específicos (por exemplo, amplitudes, latências, razão sinal/ruído e geradores de cada componente), e verificar se tais grupos fisiopatológicos correspondem a grupos psicopatológicos. Por exemplo, verificar-se-ão as correlações entre sintomatologia predominantemente negativa, de desorganização do pensamento ou paranóide, com índices de disfunção cortical em geral ou restritas ao córtex pré-frontal ou temporal.

Fonte de financiamento: Fapesp (Processos 97/11083-0 e 98/ 07640-3).

\footnotetext{
* Basile LFH, Ballester G, Castro CC, Gattaz WF. Prefrontal cortex activity assessed by high-resolution eeg and current density reconstruction. (Enviado para publicação)
} 


\section{Referências}

1. Gattaz WF, Mayer S, Ziegler P, Platz M, Gasser T. Hypofrontality on topographic EEG in schizophrenia. Eur Arch Clin Neurosci 1991;241:328-32.

2. Sponheim SR, Clementz BA, Iacono WG, Beiser M. Resting EEG in first-episode and chronic schizophrenia. Psychophysiology 1994;31(1):37-43.

3. Clementz BA, Geyer MA, Braff DL. Multiple site evaluation of P50 suppression among schizophrenia and normal comparison subjects. Schizophr Res 1998;30(1):71-80.

4. Jin Y, Potkin SG, Patterson JV, Sandman CA, Hetrick WP, Bunney WEJr. Effects of P50 temporal variability on sensory gating in schizophrenia. Psychiatry Res 1997;70(2):71-81.

5. Verleger R. Event-related potentials and cognition: A critique of the context updating hypothesis and an alternative interpretation of P3. Behavioral and Brain Sciences 1988;11:343-427.

6. Basile LFH, Rogers RL, Simos PG, Papanicolaou AC. Magnetoencephalographic evidence for common sources of long latency fields to rare target and rare novel stimuli. Int J Psychophysiol 1997;25:123-37.

7. Blackwood DH, Whalley LJ, Christie JE, Blackburn IM, St Clair DM, McInnes A. Changes in auditory P3 event-related potential in schizophrenia and depression. Br J Psychiatry 1987;150:154-60.

8. Louzã MR, Maurer K. Differences between paranoid and nonparanoid schizophrenic patients on the somatosensory P300 event-related potential. Neuropsychobiology 1989;21(2):59-66.

9. Pfefferbaum A, Ford JM, White PM, Roth WT. P3 in schizophrenia is affected by stimulus modality, response requirements, medication status, and negative symptoms. Arch Gen Psychiatry 1989;46(11):1035-44.
10.Niwa S, Hiramatsu K, Saitoh O, Fukuda M, Kameyama T, Itoh K et al. Information dysregulation and event-related potentials in schizophrenia. Schizophr Bull 1992;18(1):95-105.

11.Iwanami A, Kanamori R, Isono H, Okajima Y, Kamijima K. Impairment of inhibition of unattended stimuli in schizophrenic patients: event-related potential correlates during selective attention. Neuropsychobiology 1996;34(2):57-62.

12.Salisbury DF, O'Donnell BF, McCarley RW, Shenton ME, Benavage A. The N2 event-related potential reflects attention deficit in schizophrenia. Biol Psychol 1994;39(1):1-13.

13.Javitt DC, Doneshka P, Grochowski S, Ritter W. Impaired mismatch negativity generation reflects widespread dysfunction of working memory in schizophrenia. Arch Gen Psychiatry 1995;52(7):550-8.

14. Andrews S, Shelley AM, Ward PB, Fox A, Catts SV, McConaghy N. Event-related potential indices of semantic processing in schizophrenia. Biol Psychiatry 1993;34(7):443-58.

15.McCallum WC. Potentials related to expectancy, preparation and motor activity. In: Picton TW, editor. Handbook of Electroencephalography and Clinical Neurophysiology. Human EventRelated Potentials (revised series vol.3). Elsevier Science Publishers; 1988. p. 427-534.

16. Bachneff SA, Engelsmann F. Correlates of cerebral event-related slow potentials and psychopathology. Psychol Med 1983;13(4):763-70.

17.Wagner M, Rendtorff N, Kathmann N, Engel RR. CNV, PINV and probe-evoked potentials in schizophrenics. Electroencephalogr Clin Neurophysiol 1996;98(2):130-43.

18. Verleger R, Wascher E, Arolt V, Daase C, Strohm A, K"ompf D. Slow EEG potentials (contingent negative variation and postimperative negative variation) in schizophrenia: their association to the present state and to Parkinsonian medication effects. Clin Neurophysiol 1999;110(7):1175-92.

\section{Correspondência: Luis FH Basile}

Laboratório de Neurociências (LIM-27) - Departamento de Psiquiatria FMUSP - Av. Dr. Ovídio Pires de Campos, s/n Caixa Postal 8091 - CEP 05403-010 São Paulo, SP - Tel./fax: (0xx11) 284-6821 e 263-2201 